\title{
Histological assessment of the human heart valves and its relationship with age
}

\author{
Treerat Gumpangseth $^{1,2}$, Suree Lekawanvijit ${ }^{3}$, Pasuk Mahakkanukrauh ${ }^{2,4}$ \\ ${ }^{1} \mathrm{PhD}$ Degree Program in Anatomy, Department of Anatomy, Faculty of Medicine, Chiang Mai University, Chiang Mai, ${ }^{2}$ Department of Anatomy, \\ Faculty of Medicine, Chiang Mai University, Chiang Mai, ${ }^{3}$ Department of Pathology, Faculty of Medicine, Chiang Mai University, Chiang Mai, \\ ${ }^{4}$ Excellence in Osteology Research and Training Center (ORTC), Chiang Mai University, Chiang Mai, Thailand
}

\begin{abstract}
The human heart valves are complex anatomical structures consisting of leaflets with many supporting structures. With advancing age, the microstructure of the components of the valves can change. Knowledge and understanding of the anatomical relationships between the different components of the heart valve structures and their relationship with age is crucial for the development and progression of treatment of valvular disease. The purpose of this study was to determine histological changes of the components of the heart valves and their relationship with age. Fifty hearts taken from cadavers were included to examine the histology of the tricuspid, mitral, pulmonary, and aortic valves. All specimens were stained with Elastic Van Gieson, and picrosirius red to enable the evaluation of elastic and collagen fibers, respectively. There was a gradual increase in elastic and collagen fibers with advancing age, particularly over 40 years, in all valve types. In the case of tricuspid and mitral valves increases in collagen and elastic fibers were observed starting in the fifth decade. Elastic fiber fragmentation was observed in specimens over 50 years. In the case of the pulmonary and the aortic valves, collagen fibers were denser and more irregular in the sixth to seventh decades when compared to younger ages while elastic fibers were significantly increased in the sixth decade. In addition, an increase in fat deposition had an association with aging. These findings provide additional basic knowledge in age-related morphological changes of the heart valves and will increase understanding concerning valvular heart diseases and treatment options.
\end{abstract}

Key words: Age, Heart valves, Histology

Received April 19, 2020; Revised May 27, 2020; 2020; Accepted June 1, 2020

\section{Introduction}

Age is a significant factor in the microstructural and macrostructural changes of many body systems. In the cardiovascular system, the heart, blood vessels, and cardiac valves can change with advancing age [1-4]. Cardiovascular diseases are associated with dysfunction in the heart and vessels.

\footnotetext{
Corresponding author:

Pasuk Mahakkanukrauh (iD)

Department of Anatomy, Faculty of Medicine \& Research Cluster in Osteology Research and Training Center (ORTC), Chiang Mai University, Chiang Mai 50200, Thailand

E-mail: pasuko34@gmail.com
}

These diseases are a major cause of death worldwide in the majority of populations [5-7]. Heart valve disease is related to a high rate of mortality that can be affected by the structural or functional abnormality of the valve. Some pathological diseases of the valves often induce changes in the heart and blood vessels that can lead to heart failure. Heart valve diseases can occur in all age groups especially in older age, the incidence of disease increasing progressively after 65 years [8, 9]. This indicates that heart valve disease has a high prevalence and is life threatening. Therefore, to better understand the pathological process of the valves, a detailed knowledge of how the structures change with age in comparison to the normal condition is needed to inform intervention.

The heart valves are passive structure which are directed

\section{Copyright $\odot 2020$. Anatomy \& Cell Biology}

This is an Open Access article distributed under the terms of the Creative Commons Attribution Non-Commercial License (http://creativecommons.org/licenses/by-nc/4.0/) which permits unrestricted non-commercial use, distribution, and reproduction in any medium, provided the original work is properly cited. 
to open and close due to inertial forces exerted by the surrounding blood. They are divided into 2 main types, the atrioventricular and semilunar valves. There are specific characteristic morphological features of each valve. The tricuspid and mitral valves are the atrioventricular valves which separate the atria and the ventricles. These valves consist of three and two leaflets, respectively $[10,11]$. The semilunar valves consist of the pulmonary and aortic valves which separate the ventricles from the pulmonary artery and aorta, respectively $[1,12]$. The primary function of all the cardiac valves is maintaining the unidirectional blood flow throughout the heart although they do have different structural characteristics [2, 13]. In general, the human heart valves open and close about forty million times per year, therefore, during a lifetime they close and open over three billion times $[9,14]$. The heart pumps blood through the valves at about 3-5 liters per minute and have a highest blood velocity through the aortic valve of $1.35 \pm 0.35 \mathrm{~m} / \mathrm{s}$ [15].

Microstructural changes of the valves in relation to age are that the number of collagen fibers increase, and the parallel orientation of these fibers is lost. There are also increases in elastic fibers and a decrease in mucopolysaccharides with age. Lipid accumulation and calcification increases with advancing age, but the number of cells in the valves decrease [13, 16-20]. To date there have been few studies on histological changes with age in all valve types. Thus, the purpose of this study was to investigate the microscopic structural changes of the tricuspid, mitral, pulmonary, and aortic valves in humans and compare these findings between the valves.
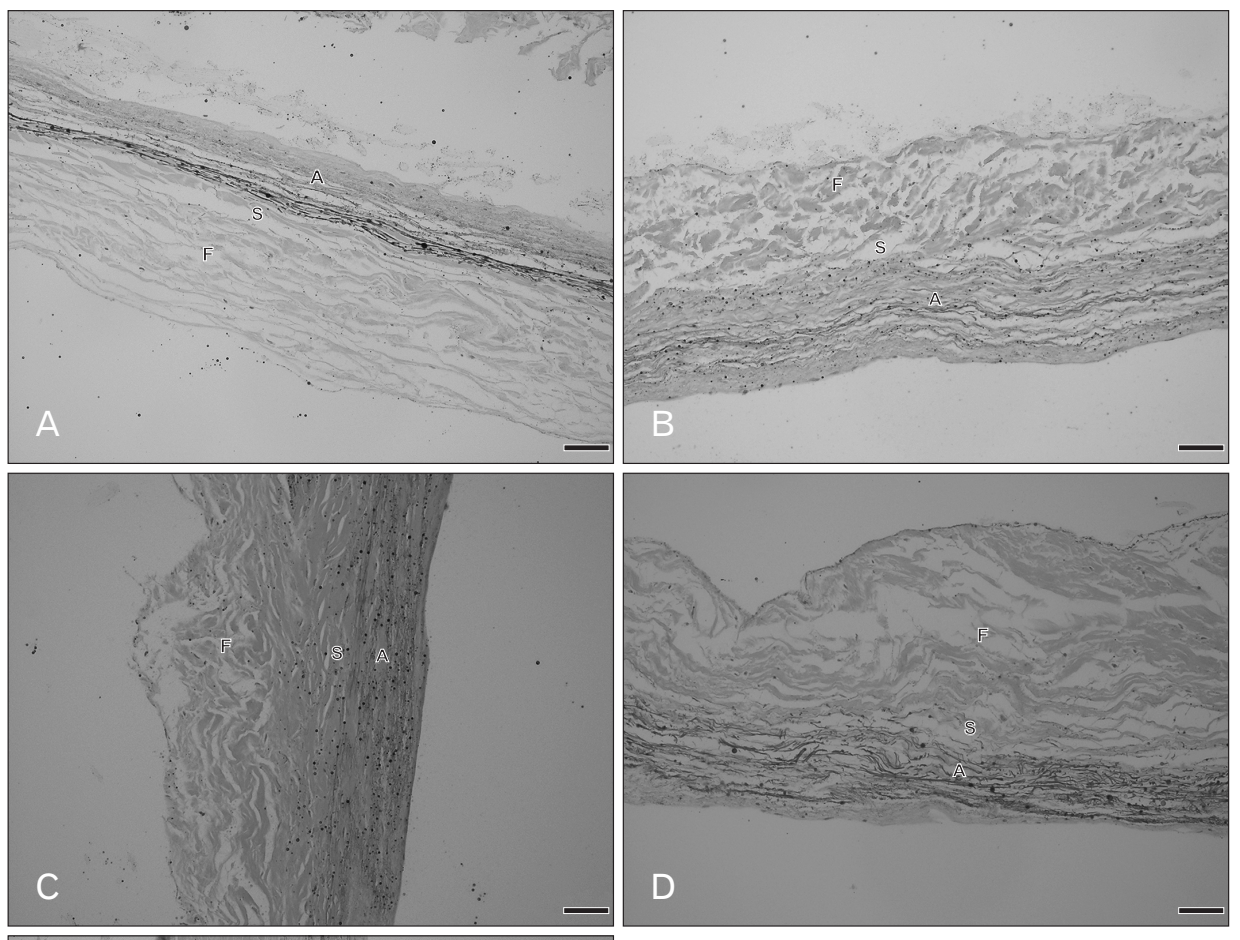

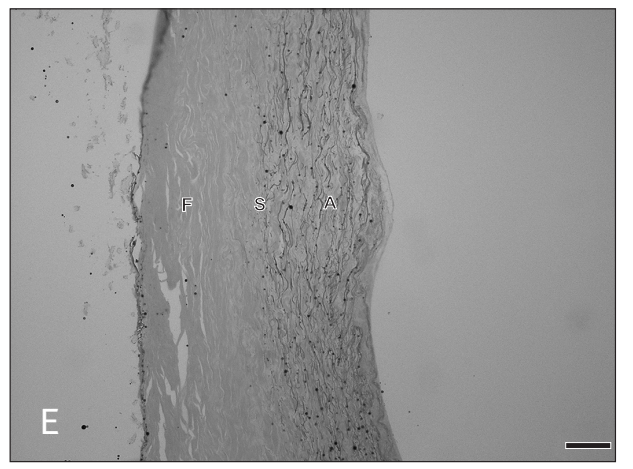

Fig. 1. Radial histological sections of the tricuspid valve in various age groups with Elastic Van Gieson staining. (A) A 20-29 years, (B) 40-49 years, (C) 50-59 years, (D) 60-69 years, (E) 70-79 years. A, atrialis; F, fibrosa; $S$, spongiosa. Scale Bars $=50 \mu \mathrm{m}(\mathrm{A}-\mathrm{E})$. 


\section{Materials and Methods}

Fifty human hearts were obtained from the donor cadavers and autopsy cases at the Faculty of Medicine, Chiang Mai University, Thailand. Individuals were aged from 20-90 years. Research on human cadaver tissues was approved by The Research Ethics Committee, Faculty of Medicine, Chiang Mai University (Research ID: ANA-2561-05232). The hearts were removed from the cadavers and cleaned, all pericardium extracts being removed from the hearts, then we dissected out all types of the heart valves. The tricuspid and mitral valve leaflets were selected from the interchordal space at the free edge of the valve attachment. The pulmonary and aortic valve leaflets were sectioned at the center area between the free edge and attachment site at the annulus. Each valve leaflet was cut from the central area and perpendicular to the base of the leaflet attachment in a radial direction. Sections were $3 \mathrm{~mm}$ wide. We investigated the histological components of the valves using picrosirius red, and Elastic Van Gieson staining. Picrosirius red was used to stain collagen fibers and elastic staining for the elastic fibers. The qualitative assessment was performed using a light microscope. Since the general morphology of each leaflet within the same valve did not differ, one leaflet of each valve was selected for examination. The anterior leaflet of the tricuspid valve, anterior leaflet of the mitral valve, anterior leaflet of the pulmonary valve, and right coronary leaflet of the aortic valve were selected for observation of the histological features.
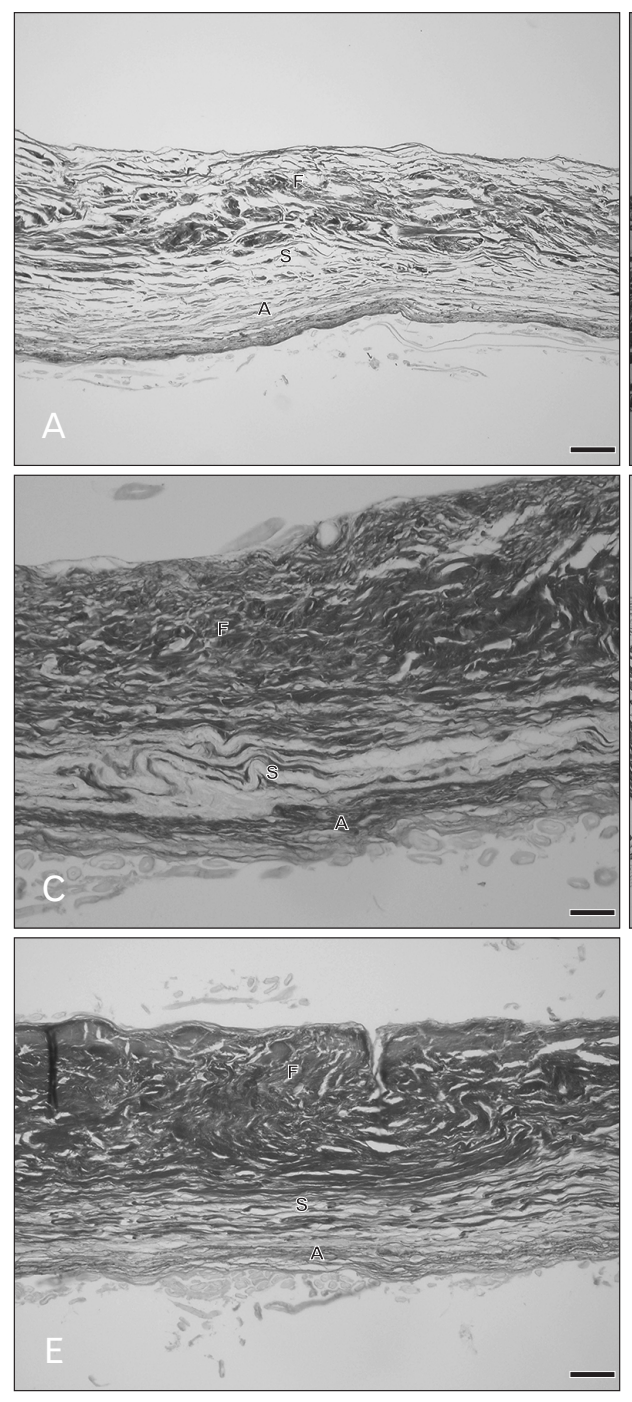
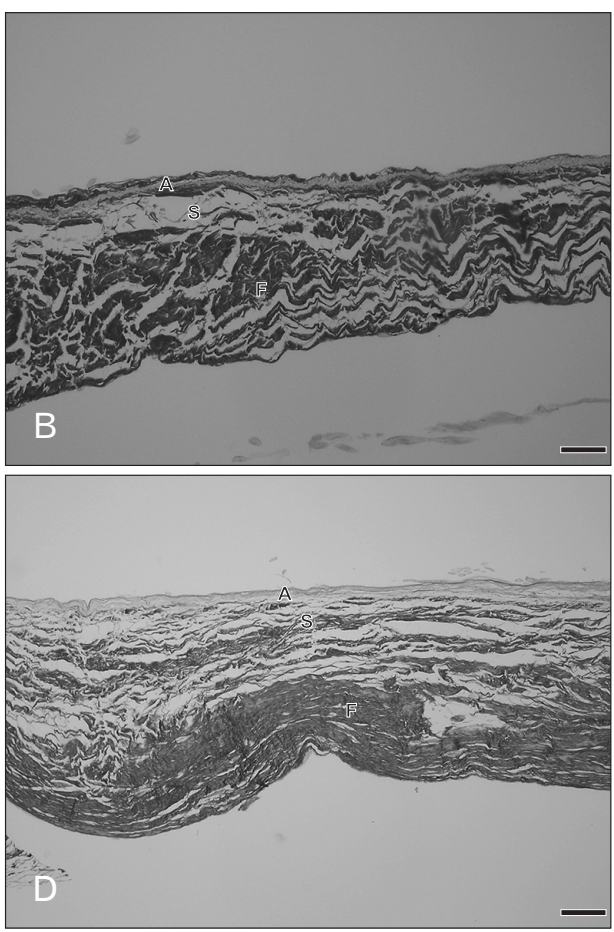

Fig. 2. Radial histological sections of the tricuspid valve in various age groups with picrosirius red staining. (A) A $20-$ 29 years, (B) 40-49 years, (C) 50-59 years, (D) 60-69 years, (E) 70-79 years. A, atrialis; F, fibrosa; $S$, spongiosa. Scale Bars $=50 \mu \mathrm{m}(\mathrm{A}-\mathrm{E})$. 


\section{Results}

\section{Tricuspid valve}

There were three distinct layers of the tricuspid valve; fibrosa, spongiosa, and atrialis. Sometimes, a ventricularis layer was also found in the tricuspid leaflet. The atrialis faced the atrium, followed by the spongiosa, fibrosa and ventricularis. The fibrosa mostly composed of collagen fibers and was the thickest layer. The spongiosa consisted of predominantly glycoaminoglycans but included loose collagen and elastic fibers. The ventricularis was the thinest layer and it sometimes could not be identified. The annulus (attachment site) and free edge region of the tricuspid valve were thicker than the middle valve region. Figs. 1 and 2 show the histological features of the tricuspid valve in cadavers of various ages. Between 20-39 years, the fibrosa showed irregular collagen bundles. Most of these fibers were arranged in a circumferential direction of the valve and the fibrosa in middle valve region was the thinnest area. The fibrosa extended continuously from the annulus and was absent at the free edge of the valve. The spongiosa had very loose collagen fibers with some elastic fibers. The atrialis consisted of very thin elastic fibers oriented parallel in a radial direction and elastic fibers were more clearly observed in the atrialis than in the ventricularis. Moreover, some collagen fibers were found in the atrialis. In heart valve from cadavers of 40-49 years, there was little fat accumulation in the free edge region and the numbers of elastic fibers gradually increased, especially in the atrialis layer. Elastic fibers were found in large numbers in the annulus and presented as multiple thin layers in a radial direction. Additionally, collagen fibers increased in number and irregularity and the spongiosa appeared narrower. In
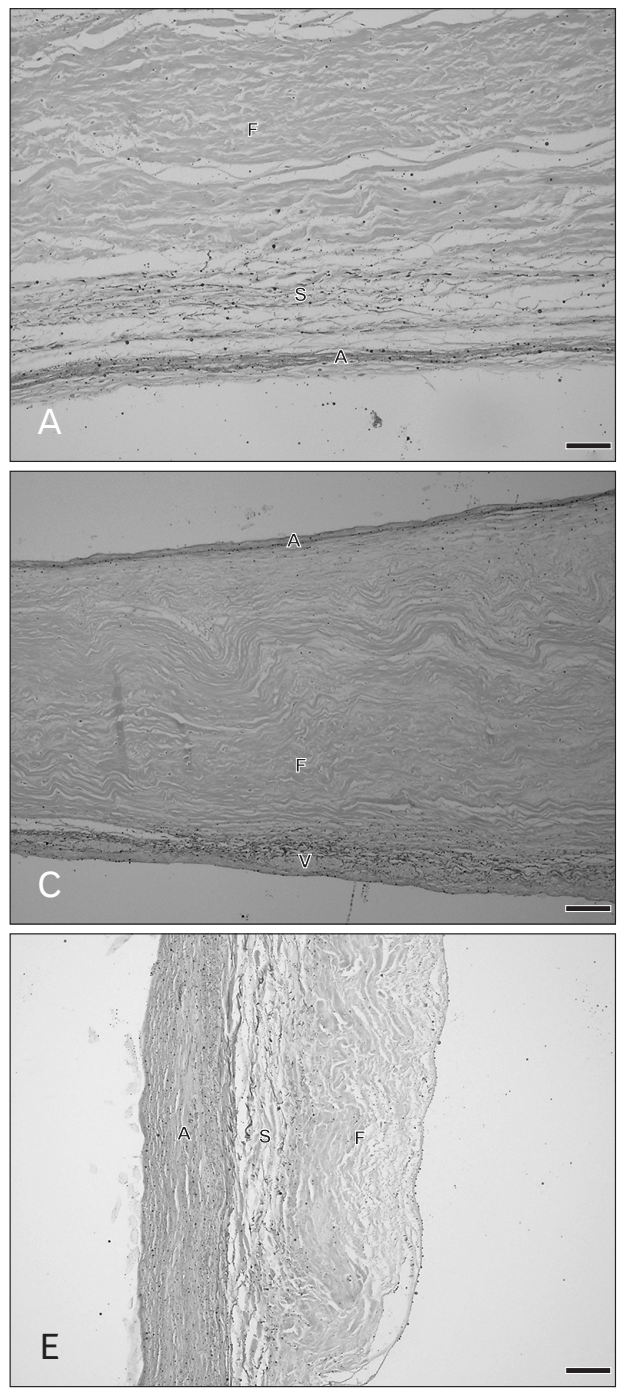
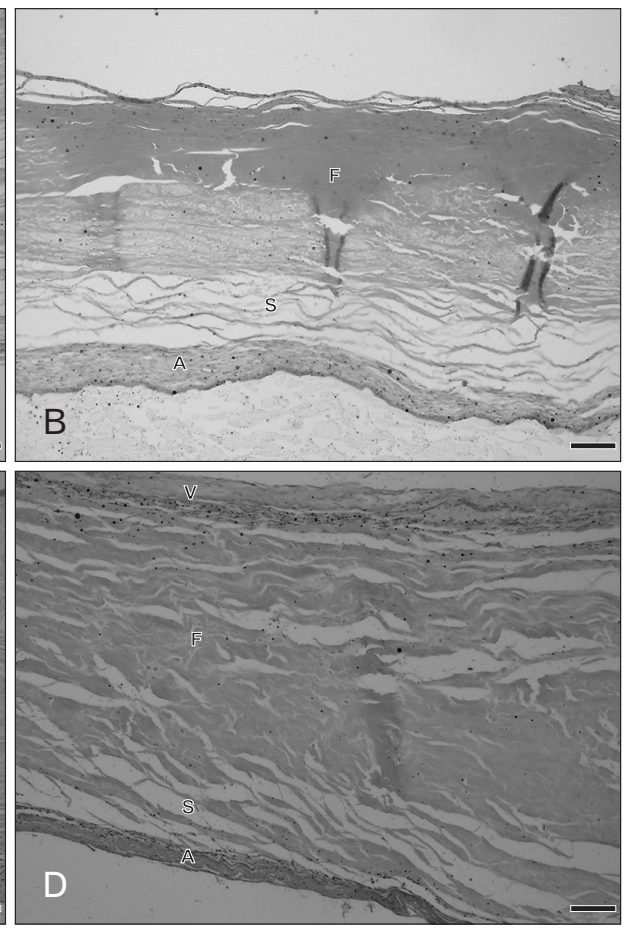

Fig. 3. Radial histological sections of the mitral valve in various age groups with Elastic Van Gieson staining. (A) A 20-29 years, (B) 40-49 years, (C) 50-59 years, (D) 60-69 years, (E) 80-89 years. A, atrialis; F, fibrosa; $S$, spongiosa; $V$, ventricularis. Scale Bars $=50 \mu \mathrm{m}(\mathrm{A}-\mathrm{E})$. 
hearts from 50-69 years, elastic fiber fragmentation and densely packed collagen fibers in the fibrosa were obvious. Over 70 years, fatty tissue presented in the tricuspid layers in some cases and elastic fibers became more fragmented and wavy. The spongiosa narrowed increasingly with age.

\section{Mitral valve}

Overall the microstructure of the mitral valve leaflet was similar to that of the tricuspid valve as were the histological changes in relation to age. These are shown in Figs. 3 and 4. At 20-39 years, the fibrosa, the thickest layer of the valve,
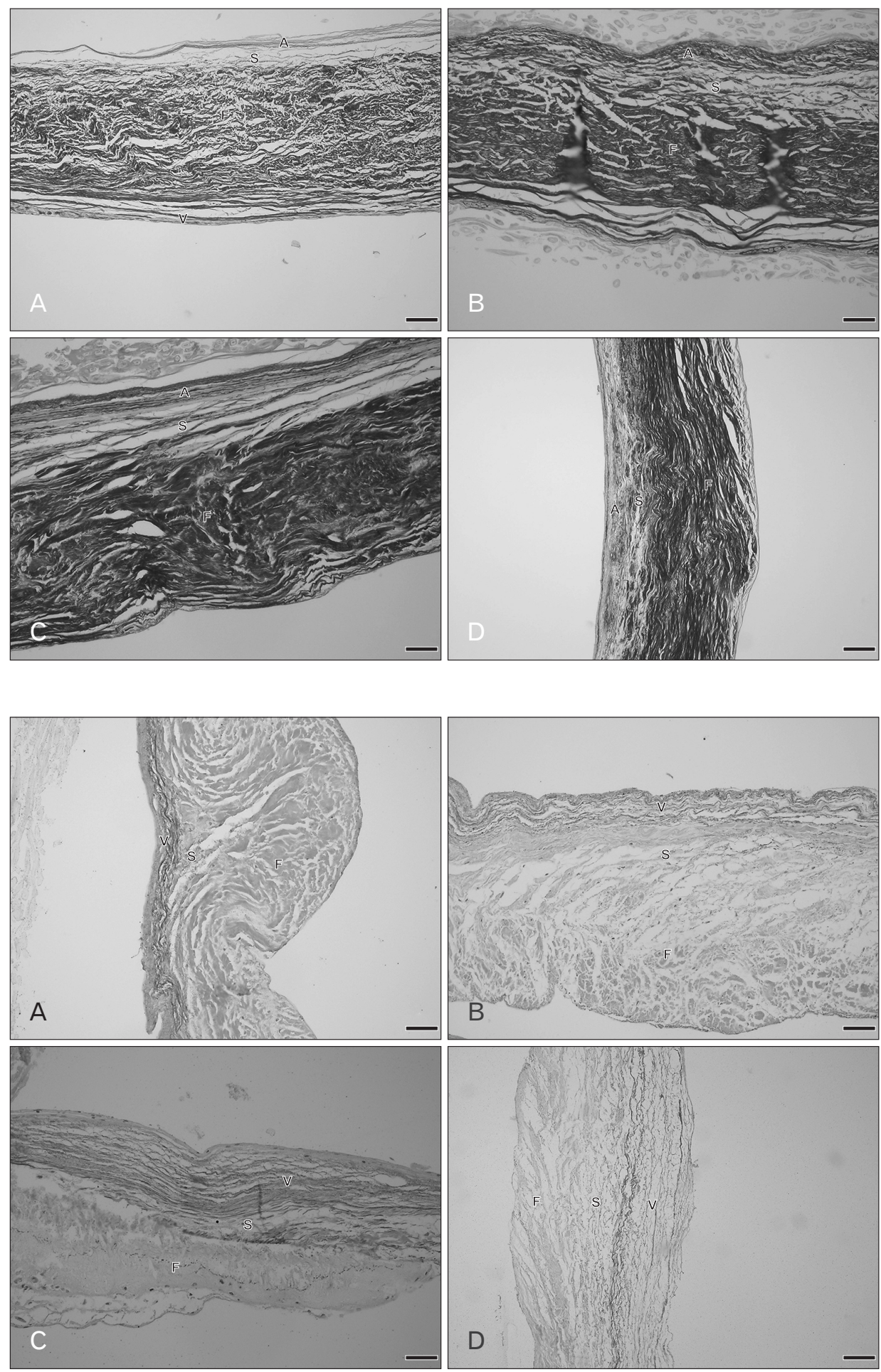

Fig. 4. Radial histological sections of the mitral valve in various age groups with picrosirius red staining. (A) A 20 29 years, (B) 40-49 years, (C) 60-69 years, (D) 80-89 years. A, atrialis; F, fibrosa; $S$, spongiosa; $V$, ventricularis. Scale Bars $=50 \mu \mathrm{m}(\mathrm{A}), 20 \mu \mathrm{m}(\mathrm{B}-\mathrm{D})$.

Fig. 5. Radial histological sections of the pulmonary valve in various age groups with Elastic Van Gieson staining. (A) A 30-39 years, (B) 50-59 years, (C) 60-69 years, (D) 70-79 years. F, fibrosa; $S$, spongiosa; $V$, ventricularis. Scale Bars $=50 \mu \mathrm{m}(\mathrm{A}-\mathrm{D})$. 
consisted of irregular collagen bundles mostly arranged in a circumferential direction especially in the central region. However some collagen fibers in the fibrosa were arranged in a radial direction. In both the atrialis and ventricularis, multiple thin layers of elastic fibers and some collagen fibers were observed. The atrialis contained more elastic fibers than the ventricularis. The spongiosa showed loose connective tissue. No remarkable changes of elastic fibers were observed in this age period. The annulus site (attachment site) of the mitral valve was thicker than the free edge and fat deposits were initially seen in both the annulus and free edge in the age group 30-39 years. In hearts from cadavers aged 40-49 years, more fat acummulation was found in the annulus and the collagen fibers in the fibrosa became denser. There were a slight increase in numbers of collagen and elastic fibers and the elastic fibers being initially fragmented. In the ventricu- laris of the mitral leaflet was more identifiable elastic fibers were present than in the tricuspid valve especially in the free edge. In the 50-69 year hearts, elastic fiber fragmentation in the atrialis was obvious and thin layers of elastic fibers were still seen in the ventricularis. The numbers of collagen and elastic fibers gradually increased with age. Collagen fiber arrangement in the fibrosa was more irregular and denser in this age period. In addition, there was fat deposition between the valve layers. In 70-79 year hearts, changes were similar. Over 80 years, elastic fibers clearly increased in the atrialis layer particulary on the annulus side. The proportions of both collagen and elastic fibers in this age group were at their highest with the thickest fibrosa and thinest spongiosa.

\section{Pulmonary valve}

Figs. 5 and 6 show the histology of the pulmonary valves
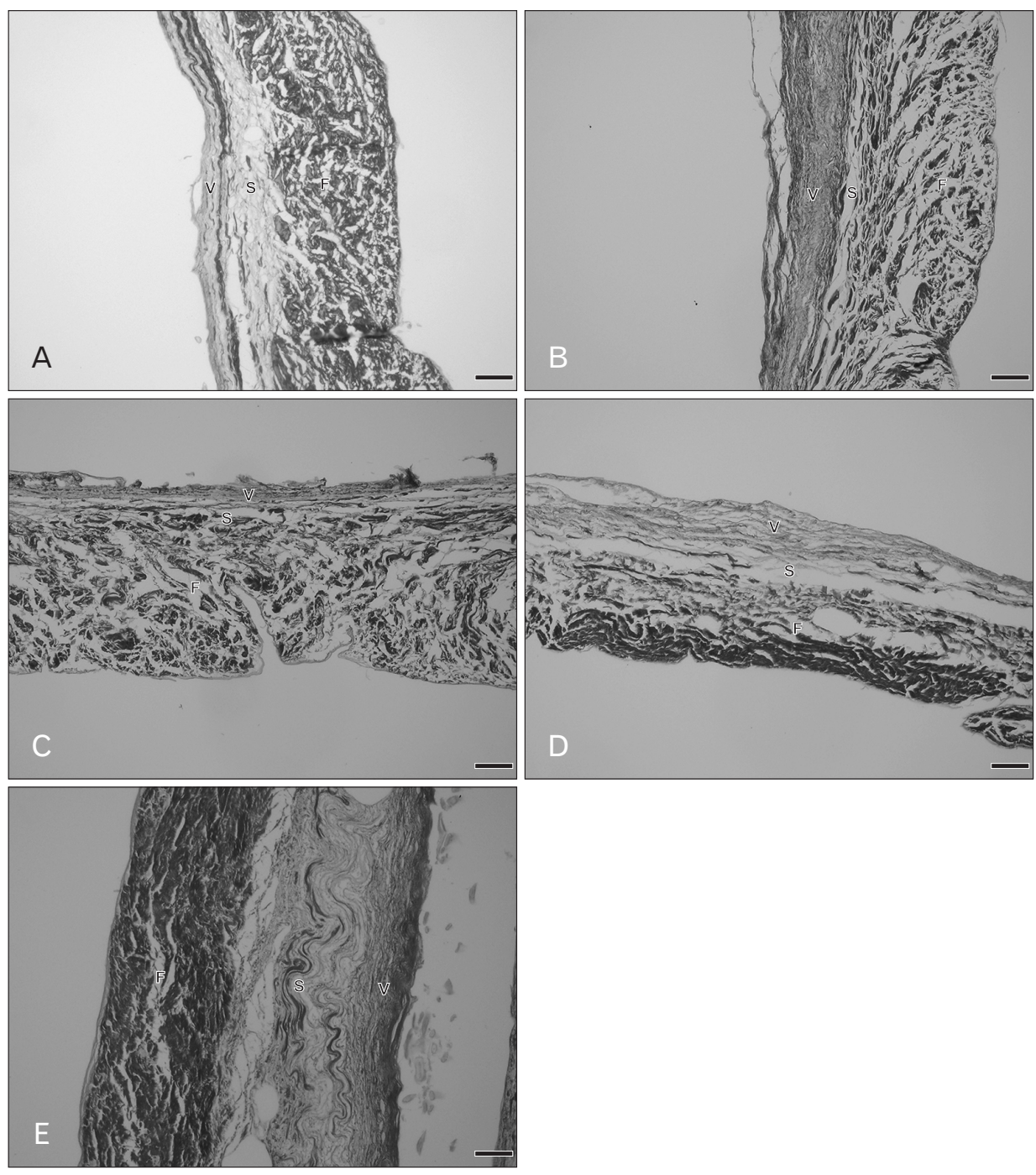

Fig. 6. Radial histological sections of the pulmonary valve in various age groups with picrosirius red staining. (A) A 2029 years, (B) 40-49 years, (C) 50-59 years, (D) 60-69 years, (E) 70-79 years. $\mathrm{F}$, fibrosa; $\mathrm{S}$, spongiosa; $\mathrm{V}$, ventricularis. Scale Bars $=20 \mu \mathrm{m}$ (A), $50 \mu \mathrm{m}$ (B-E). 
with three distinct layers: fibrosa, spongiosa, and ventricularis. The spongiosa is situated between the fibrosa and the ventricularis. The free edge was thicker than the annulus side. In the 20-39 year hearts, irregular collagen fibers were found in the fibrosa. The spongiosa showed loose collagen fibers oriented parallel in a circumferential direction and this layer was largest in the younger age group. Thin parallel collagen fibers were observed in the ventricularis. There were thin layers of elastic fibers in the ventricularis and this layer was obvious in the semilunar valves. Fatty tissue was not observed in the pulmonary leaflet in this age group. There were no remarkable changes in the elastic fibers in the 20-49 year age group while there are slight increases in collagen fibers. At 50-69 years, the numbers of collagen fibers were increased and the orientation of the fibers was more irregular and denser. Elastic fibers were also obviously increasing with increasing age. Over 60 years, fatty tissues were found within the valve leaflet. An increase in denser collagen fibers in age over 70 years was observed in all valve layers. Most elastic fibers showed fragmentation in the ventricularis.

\section{Aortic valve}

The general histological features of the aortic valve were similar to the pulmonary valve. Figs. 7 and 8 demonstrate the histological changes with age of the aortic valves. At age 20-39 years, the fibrosa mostly contained dense and irregular collagen fibers arranged in a circumferential direction. The spongiosa was the thickest and the ventricularis was the thinnest. Thin collagen fibers oriented in a radial direction were found in the ventricularis. More and denser collagen fibers were present in the fibrosa of the aortic valve compared with pulmonary valve. The proportion of elastic fibers
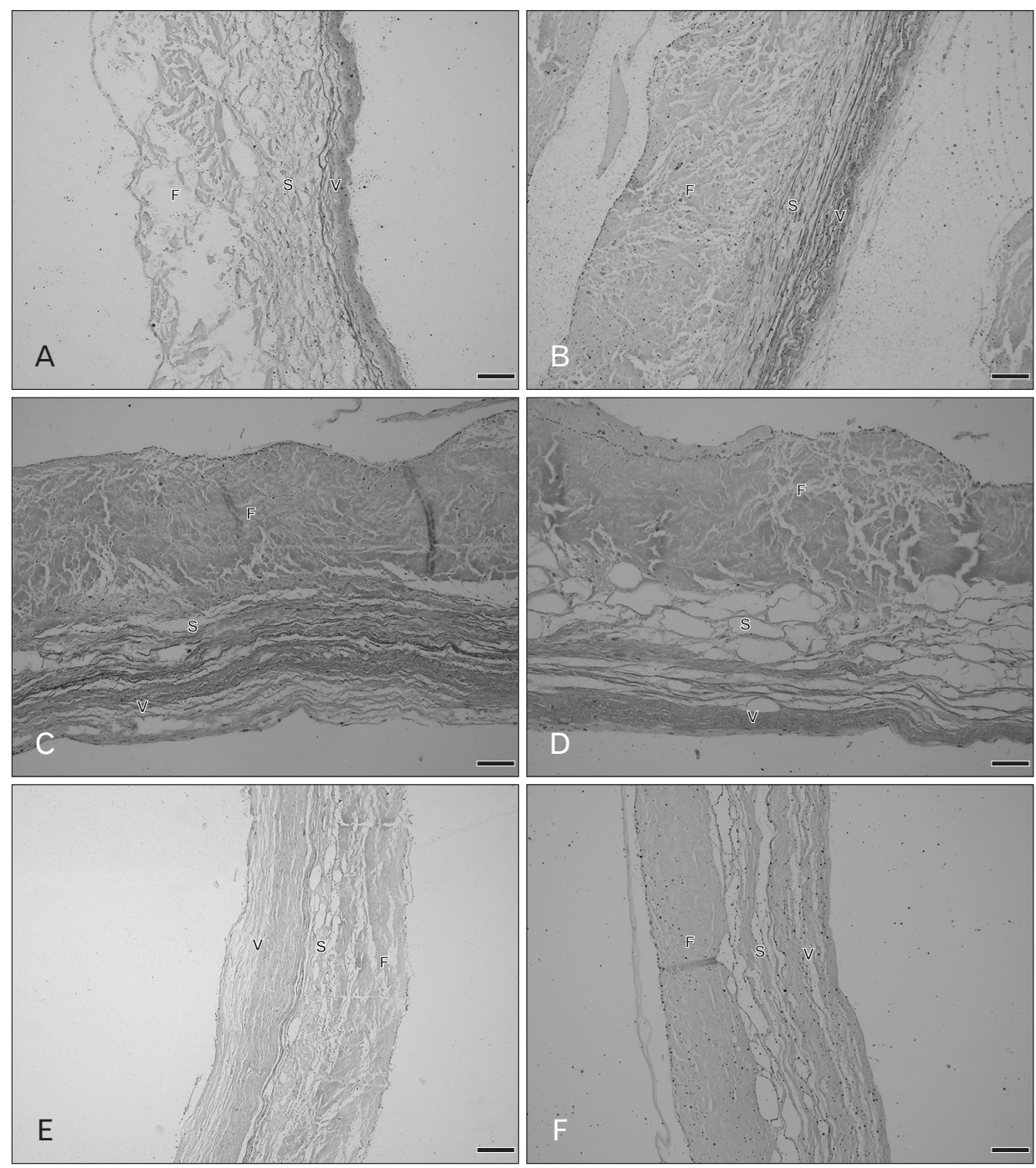

Fig. 7. Radial histological sections of the aortic valve in various age groups with Elastic Van Gieson staining. (A) A 20-29 years, (B) 40-49 years, (C) 50-59 years, (D) 60-69 years, (E) 70 79 years, (F) 80-89 years. F, fibrosa; $\mathrm{S}$, spongiosa; $\mathrm{V}$, ventricularis. Scale Bars $=50 \mu \mathrm{m}(\mathrm{A}-\mathrm{F})$. 

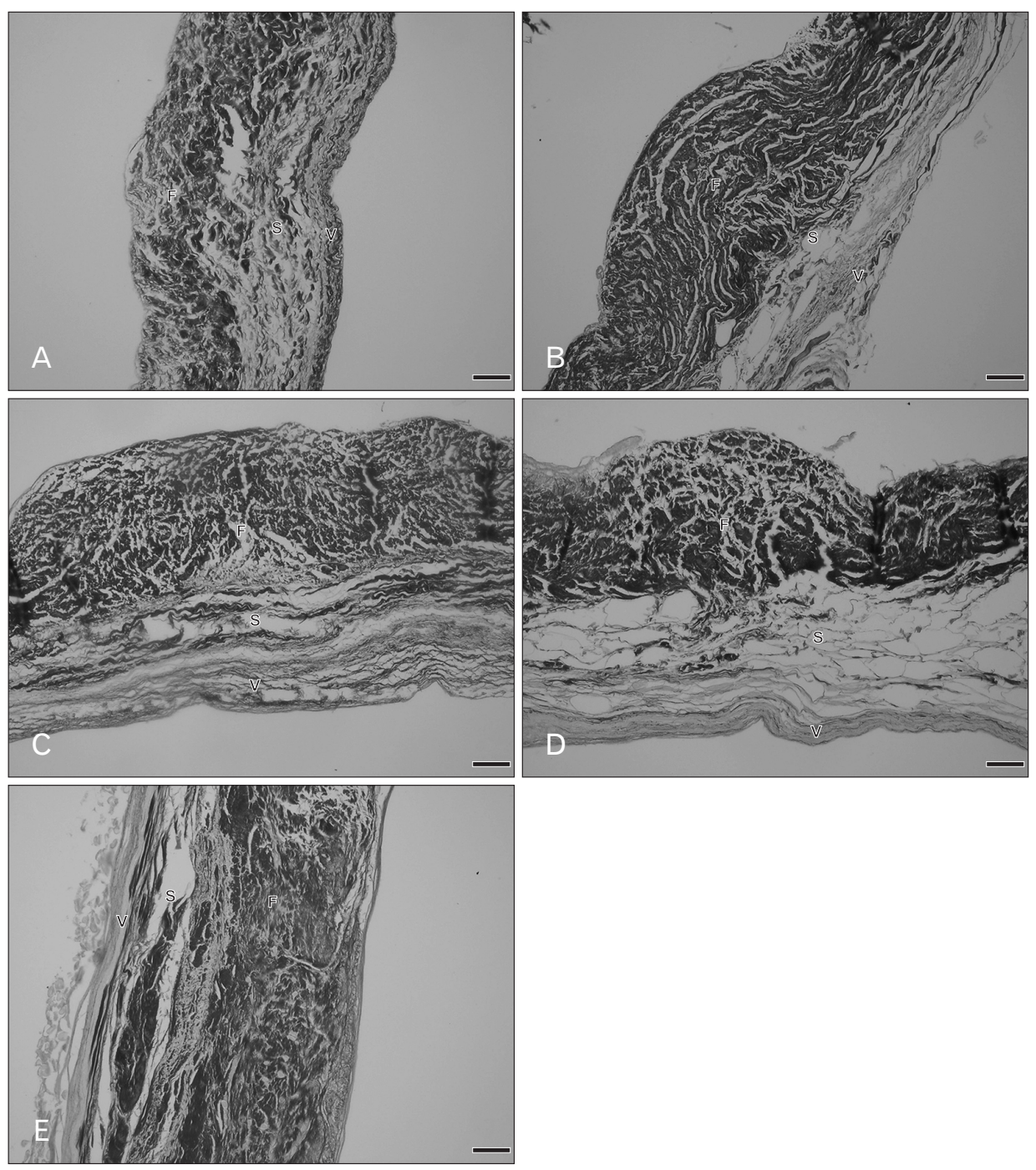

Fig. 8. Radial histological sections of the aortic valve in various age groups with picrosirius red staining. (A) A 20 29 years, (B) 40-49 years, (C) 50-59 years, (D) 60-69 years, (E) 70-79 years. $\mathrm{F}$, fibrosa; $\mathrm{S}$, spongiosa; $\mathrm{V}$, ventricularis. Scale Bars $=100 \mu \mathrm{m}(\mathrm{A}), 50 \mu \mathrm{m}(\mathrm{B}-\mathrm{E})$.

appeared to be highest in the aortic valve compared with other valves. In hearts of age over 30 years there was little fat accumulation at the annulus side. In hearts from 40-59 year old cadavers the spongiosa appeared narrower and the fibrosa was thicker. Collagen and elastic fibers gradually increased with increasing age. By 50-59 years, elastic fibers were clearly increasing. Over 60 years, an increase in dense and irregular collagen fibers were obvious in the aortic leaflet. The fibrosa was the thickest layer and there was more fat accumulation within aortic leaflets. In 70-90 years, elastic fibers were substantially increased in the venticularis and its thickness increased to that of the fibrosa.

\section{Discussion}

In this study the histological features of distinct layers of the tricuspid, mitral, pulmonary, and aortic valves are presented. The heart valves consist of different layers which have various types of extracellular matrix (ECM) and these components provide high tension strength to offer resistance to transvalvular pressures during the cardiac cycle for optimal function $[4,21]$. The fibrosa composed of mostly dense packed of collagen bundles and spongiosa had few loose collagen and elastic fibers. The ventricularis was thinnest layer contained the parallel elastic fibers whereas the atrialis was clearly seen in the atrioventricular valves (tricuspid and mitral valves). We studied the histological changes of the heart valves especially in collagen and elastic fibers with age. Collagen fibers were mainly containing in the fibrosa comprised the strongest portion of the valve leaflet that was responsible for bearing diastolic stress. It provided the stiffness and limit extensibility of the valve tissue. We also found that the 
fibrosa not extend along the whole thickness of the valve and absented at the free edge of all valve leaflets. Conversely, elastic fibers in the atrialis and ventricularis were high elasticity structure and low stiffness that provided the ability to extension and recoil to the original shape $[1,4]$. Our findings indicated that aging was accompanied by changes of ECM like collagen and elastic structures.

We examined the histology of all valve types between $20-$ 90 years and found that there were progressively changes in the microstructural compositions of the valve relation to age. For the tricuspid valve, there were seen most dense collagen fibers in the fibrosa. Elastic fibers were observed in radial direction of the valve. The ventricularis was not clearly seen in this valve and it seem that the tricuspid valve had more wavy elastic fibers among other valves. In 40-49 years, collagen and elastic fibers showed gradually increased with increasing age Then, the elastic fibers were more fragmented with denser collagen fibers above 50 years. In the mitral valve, the compositions were similar to the tricuspid valve. There were gradually increased of collagen fibers and presented elastic fibers fragmentations in 40-49 years. Elastic fibers in the atrialis were clearly observed in the mitral leaflet. Beyond 50 years, collagen fibers orientation was more irregular and denser with more fragmented of elastic fibers. The fibrosa was thicker with increasing age and thickest in over 80 years. Changing of both tricuspid and mitral valves was similar however, the mitral valve was thicker with high components than the tricuspid valve. Our results were consistent with others which reported by Sell and Scully [22], the qualitative study found that for second to fifth decades of the mitral valve, the collagen fibers presented thicker and not parallel arrangement and was slightly increasing of the elastic fibers. The annulus had lipid accumulation in third decade and more deposited in 40 years. Over 50 years the collagen fibers were more disorientated. In this study, we also found little fat deposition in the annulus and free edge in aged 30-39 years and beyond 50 years fat deposition more increased within the mitral leaflet. While there was found fatty tissue deposition in the tricuspid valves over 40 years.

The pulmonary and aortic valves were similar histological morphology but there was the difference of changing with age. In both semilunar valves, showed clearly thin layer of elastic fibers in the ventricularis. Over 40 years collagen and elastic fibers were gradually increased with age and the fibrosa was thicker beyond 60 years. The aortic valves were thicker than pulmonary leaflets in both gross and histologi- cal examination including fat deposition within the aortic leaflets over 30 years. Some cases of the pulmonary valves found fatty tissue over 60 years.

Most of previous studies investigated the histology of the pulmonary and aortic valves. They studied in age between fetus to adults and found most histological changes in fetus to adolescent group. Earlier studies determined microstructures of the normal aortic and pulmonary valves in various age groups. They divided samples into many groups as fetus in the second trimester (14-19 weeks), fetus in the third trimester (20-39 weeks), early neonatal (minutes to hours), late neonatal (up to 30 days), children ( $6.0 \pm 1.6$ years), adults $(50.1 \pm 2.5$ years). They used the image analysis software to quantify the percentages of each component within the valves. The results showed that at 14-19 weeks of fetus there was no distinguish into layer and composed of most glycosaminoglycans and low density of collagen and elastic fibers. At 20-39 weeks of fetus, there were presented elastic fibers in the ventricularis and collagen fibers were increased in the fibrosa. The results showed the trilaminar structure was presented at 36 weeks of fetus and collagen fibers increased in fetus and unchanged. Moreover, there was increasing elastic fibers with advancing age. When compared to the aortic valves, the pulmonary valve had less elastic fibers in adult groups [20]. Another study done by van Geemen et al. [18] investigated age-related changes on morphology, microstructures, and biomechanical properties of the human aortic and pulmonary valves. They divided samples into 3 age groups as follows: 2 months-11 years, $18-22$ years, and 35-53 years. Histological results showed collagen fibers were increased with age. There were no different between the pulmonary and aortic valves for histological compositions [18].

Additionally, the other studies have also shown that there was increasing accumulation of lipid in the aortic valve leaflets with advancing age. The lipid found in the fibrosa over 30 years [16]. Our results also found initially fat accumulation in the aortic valve leaflets in fourth decade (30-39 years) in annulus whereas found in seventh decade (60-69 years) of the pulmonary valves. The collagen and elastic fibers in the tricuspid valve were seen to be not as tightly arrangement along the radial direction as other valves that may resulting from the different morphology and biomechanics function of each valve types. However, the histology of the aortic valves seemed similar to the mitral valve. The collagen fibers were densely packed, and the thickness was similar. Four heart valves are opening and closing directed by blood flow 
in different pressure conditions in each side of the heart. Blood flow from the atria which have low pressure into the ventricles that is high pressure. The left side has higher pressure than in the right side of the heart so the thickness between these valves was also different $[1,21]$. The physiological transvalvular pressures in normal condition of the heart valves were $25 \mathrm{mmHg}$ of the tricuspid, $10 \mathrm{mmHg}$ of the pulmonary, $120 \mathrm{mmHg}$ of the mitral, and $80 \mathrm{mmHg}$ of the aortic valves [23]. These might be caused similarity changes between the mitral and aortic valves.

Since age is the one of risk factors for valvular diseases, some disease occurs progressively with age especially the left side of the heart [24-26]. Calcific changes can occur in aging valve. Calcification of the valve was calcium deposits. The valve leaflets became stiffer and presented nodular calcifications in the annulus and valve surface and this condition interrupted organization of the collagen fibers. Histological examination demonstrated calcified masses within the leaflets that ultimately protrude through the outflow surfaces [24, 27-29]. We found larger calcific nodule in the older specimens especially in the aortic valve and found mostly in age over 70 years. However, early stage of valve calcification was characterized by yellowish area (lipid accumulation and fibrosis) without calcific nodule. Limitations of this study were small sample sizes and the difference between male and female need to be considered. Most previous literatures studied the morphology of the valves related with age in fetus, child, and adolescent. Suggesting that the morphology of the heart valves was clearly and rapid changed in fetus to adolescent rather than in adult and older groups. In current study, we found gradually changes of the valve structures with advancing age and age-related changes range was rather wide. However, knowledge of the heart valve microstructures dependent with age in normal was crucial for valve disease conditions.

\section{ORCID}

Treerat Gumpangseth:

https://orcid.org/0000-0003-0625-5023

Suree Lekawanvijit:

https://orcid.org/0000-0002-5488-8119

Pasuk Mahakkanukrauh:

https://orcid.org/0000-0003-0611-7552

\section{Author Contributions}

Conceptualization: TG, SL, PM. Data acquisition: TG. Data analysis or interpretation: TG. Drafting of the manuscript: TG. Critical revision of the manuscript: TG, SL, PM. Approval of the final version of the manuscript: all authors.

\section{Conflicts of Interest}

No potential conflict of interest relevant to this article was reported.

\section{Acknowledgements}

This study was funded by the Faculty of Medicine, Chiang Mai University, Chiang Mai, Thailand (Research ID: ANA2561-05232). We would also like to thank the Excellence in Osteology Research and Training Center (ORTC) with partially supported by Chiang Mai University.

\section{References}

1. Hinton RB, Yutzey KE. Heart valve structure and function in development and disease. Annu Rev Physiol 2011;73:29-46.

2. Misfeld M, Sievers HH. Heart valve macro- and microstructure. Philos Trans R Soc Lond B Biol Sci 2007;362:1421-36.

3. Anderson RH, Ho SY, Becker AE. Anatomy of the human atrioventricular junctions revisited. Anat Rec 2000;260:81-91.

4. Mendelson K, Schoen FJ. Heart valve tissue engineering: concepts, approaches, progress, and challenges. Ann Biomed Eng 2006;34:1799-819.

5. Mozaffarian D, Benjamin EJ, Go AS, Arnett DK, Blaha MJ, Cushman M, Das SR, de Ferranti S, Després JP, Fullerton HJ, Howard VJ, Huffman MD, Isasi CR, Jiménez MC, Judd SE, Kissela BM, Lichtman JH, Lisabeth LD, Liu S, Mackey RH, Magid DJ, McGuire DK, Mohler ER 3rd, Moy CS, Muntner P, Mussolino ME, Nasir K, Neumar RW, Nichol G, Palaniappan L, Pandey DK, Reeves MJ, Rodriguez CJ, Rosamond W, Sorlie PD, Stein J, Towfighi A, Turan TN, Virani SS, Woo D, Yeh RW, Turner MB. Heart Disease and Stroke Statistics-2016 update: a report from the American Heart Association. Circulation 2016;133:e38-360.

6. Benjamin EJ, Blaha MJ, Chiuve SE, Cushman M, Das SR, Deo R, de Ferranti SD, Floyd J, Fornage M, Gillespie C, Isasi CR, Jiménez MC, Jordan LC, Judd SE, Lackland D, Lichtman JH, Lisabeth L, Liu S, Longenecker CT, Mackey RH, Matsushita K, Mozaffarian D, Mussolino ME, Nasir K, Neumar RW, Palaniappan L, Pandey DK, Thiagarajan RR, Reeves MJ, Ritchey M, Rodriguez CJ, Roth GA, Rosamond WD, Sasson C, Towfighi A, Tsao CW, Turner MB, Virani SS, Voeks JH, Willey JZ, 
Wilkins JT, Wu JH, Alger HM, Wong SS, Muntner P. Heart Disease and Stroke Statistics-2017 update: a report from the American Heart Association. Circulation 2017;135:e146-603.

7. Nichols M, Townsend N, Scarborough P, Rayner M. Cardiovascular disease in Europe: epidemiological update. Eur Heart J 2013;34:3028-34.

8. Iung B, Vahanian A. Epidemiology of valvular heart disease in the adult. Nat Rev Cardiol 2011;8:162-72.

9. Schoen FJ. Morphology, clinicopathologic correlations, and mechanisms in heart valve health and disease. Cardiovasc Eng Technol 2018;9:126-40.

10. Athavale S, Deopujari R, Sinha U, Lalwani R, Kotgirwar S. Is tricuspid valve really tricuspid? Anat Cell Biol 2017;50:1-6.

11. Gupta C, Shetti VR, Manju BVM. Dimensions of the human adult mitral valve in the embalmed cadaver. J Morphol Sci 2013;30:6-10.

12. Gross L, Kugel MA. Topographic anatomy and histology of the valves in the human heart. Am J Pathol 1931;7:445-74.7.

13. Pham T, Sulejmani F, Shin E, Wang D, Sun W. Quantification and comparison of the mechanical properties of four human cardiac valves. Acta Biomater 2017;54:345-55.

14. Parvin Nejad S, Blaser MC, Santerre JP, Caldarone CA, Simmons CA. Biomechanical conditioning of tissue engineered heart valves: too much of a good thing? Adv Drug Deliv Rev 2016;96:161-75.

15. Hasan A, Ragaert K, Swieszkowski W, Selimović S, Paul A, Camci-Unal G, Mofrad MR, Khademhosseini A. Biomechanical properties of native and tissue engineered heart valve constructs. J Biomech 2014;47:1949-63.

16. Kim KM, Valigorsky JM, Mergner WJ, Jones RT, Pendergrass RF, Trump BF. Aging changes in the human aortic valve in relation to dystrophic calcification. Hum Pathol 1976;7:47-60.

17. Balguid A, Rubbens MP, Mol A, Bank RA, Bogers AJ, van Kats JP, de Mol BA, Baaijens FP, Bouten CV. The role of collagen cross-links in biomechanical behavior of human aortic heart valve leaflets--relevance for tissue engineering. Tissue Eng 2007;13:1501-11.

18. van Geemen D, Soares AL, Oomen PJ, Driessen-Mol A,
Janssen-van den Broek MW, van den Bogaerdt AJ, Bogers AJ, Goumans MJ, Baaijens FP, Bouten CV. Age-dependent changes in geometry, tissue composition and mechanical properties of fetal to adult cryopreserved human heart valves. PLoS One 2016;11:e0149020.

19. McDonald PC, Wilson JE, McNeill S, Gao M, Spinelli JJ, Rosenberg F, Wiebe H, McManus BM. The challenge of defining normality for human mitral and aortic valves: geometrical and compositional analysis. Cardiovasc Pathol 2002;11:193209.

20. Aikawa E, Whittaker P, Farber M, Mendelson K, Padera RF, Aikawa M, Schoen FJ. Human semilunar cardiac valve remodeling by activated cells from fetus to adult: implications for postnatal adaptation, pathology, and tissue engineering. Circulation 2006;113:1344-52.

21. Sacks MS, David Merryman W, Schmidt DE. On the biomechanics of heart valve function. J Biomech 2009;42:1804-24.

22. Sell S, Scully RE. Aging changes in the aortic and mitral valves. Histologic and histochemical studies, with observations on the pathogenesis of calcific aortic stenosis and calcification of the mitral annulus. Am J Pathol 1965;46:345-65.

23. Guyton AC. Textbook of medical physiology. 8th ed. Philadelphia: Saunders; 1986.

24. Waller BF. The old-age heart: normal aging changes which can produce or mimic cardiac disease. Clin Cardiol 1988;11:513-7.

25. Pomerance A. Ageing changes in human heart valves. Br Heart J 1967;29:222-31.

26. Donnelly KB. Cardiac valvular pathology: comparative pathology and animal models of acquired cardiac valvular diseases. Toxicol Pathol 2008;36:204-17.

27. Somers P, Knaapen M, Mistiaen W. Histopathology of calcific aortic valve stenosis. Acta Cardiol 2006;61:557-62.

28. Angelini A, Basso C, Grassi G, Casarotto D, Thiene G. Surgical pathology of valve disease in the elderly. Aging (Milano) 1994;6:225-37.

29. Warren BA, Yong JL. Calcification of the aortic valve: its progression and grading. Pathology 1997;29:360-8. 